\title{
A RESULT RELATED TO DERIVATIONS ON UNITAL SEMIPRIME RINGS
}

\author{
Irena Kosi-Ulbl, NejC Širovnik and Joso Vukman \\ University of Maribor, Slovenia
}

\begin{abstract}
The purpose of this paper is to prove the following result. Let $n \geq 3$ be some fixed integer and let $R$ be a $(n+1) ! 2^{n-2}$-torsion free semiprime unital ring. Suppose there exists an additive mapping $D: R \rightarrow$ $R$ satisfying the relation

$2^{n-2} D\left(x^{n}\right)$

$$
\begin{aligned}
= & \left(\sum_{i=0}^{n-2}\left(\begin{array}{c}
n-2 \\
i
\end{array}\right) x^{i} D\left(x^{2}\right) x^{n-2-i}\right)+\left(2^{n-2}-1\right)\left(D(x) x^{n-1}+x^{n-1} D(x)\right) \\
& +\sum_{i=1}^{n-2}\left(\sum_{k=2}^{i}\left(2^{k-1}-1\right)\left(\begin{array}{c}
n-k-2 \\
i-k
\end{array}\right)+\sum_{k=2}^{n-1-i}\left(2^{k-1}-1\right)\left(\begin{array}{c}
n-k-2 \\
n-i-k-1
\end{array}\right)\right)
\end{aligned}
$$$$
x^{i} D(x) x^{n-1-i}
$$

for all $x \in R$. In this case $D$ is a derivation. The history of this result goes back to a classical result of Herstein, which states that any Jordan derivation on a 2 -torsion free prime ring is a derivation.
\end{abstract}

Throughout, $R$ will represent an associative ring with center $Z(R)$. Given an integer $n>1$, a ring $R$ is said to be $n$-torsion free if for $x \in R, n x=0$ implies $x=0$. As usual, the commutator $x y-y x$ will be denoted by $[x, y]$. Recall that a ring $R$ is prime if for $a, b \in R, a R b=(0)$ implies either $a=0$ or $b=0$ and is semiprime in case $a R a=(0)$ implies $a=0$.

An additive mapping $D: R \rightarrow R$, where $R$ is an arbitrary ring, is called a derivation if $D(x y)=D(x) y+x D(y)$ holds for all pairs $x, y \in R$. A derivation is inner in case there exists $a \in R$ such that $D(x)=[a, x]$ holds for all $x \in R$. An additive mapping $D: R \rightarrow R$ is called a Jordan derivation in case $D\left(x^{2}\right)=D(x) x+x D(x)$ is fulfilled for all $x \in R$. Every derivation is a

2020 Mathematics Subject Classification. 16N60, 39B52.

Key words and phrases. Prime ring, semiprime ring, derivation, Jordan derivation. 
Jordan derivation. The converse is in general not true. A classical result of Herstein ([13]) asserts that any Jordan derivation on a 2-torsion free prime ring is a derivation. A brief proof of Herstein theorem can be found in [3]. Cusack ([6]) has generalized Herstein theorem to 2-torsion free semiprime rings (see [4] for an alternative proof). Beidar, Brešar, Chebotar and Martindale ([2]) have fairly generalized Herstein theorem.

Brešar ([5]) has proved the following result (see [16] for a generalization).

TheOREM 1. Let $R$ be a 2-torsion free semiprime ring and let $D: R \rightarrow R$ be an additive mapping satisfying the relation

$$
D(x y x)=D(x) y x+x D(y) x+x y D(x)
$$

for all $x, y \in R$. In this case $D$ is a derivation.

An additive mapping $D$, which maps an arbitrary ring $R$ into itself and satisfies the relation (1) for all pairs $x, y \in R$, is called a Jordan triple derivation. One can easily prove that any Jordan derivation on an arbitrary 2-torsion free ring is a Jordan triple derivation, which means that Theorem 1 generalizes Cusack's generalization of Herstein theorem.

The above result represents a motivation for many other results (for example $[10,14,17])$. Vukman $([18])$ conjectured that in case there exists an additive mapping $D: R \rightarrow R$, where $R$ is a 2-torsion free semiprime ring, satisfying the relation

$$
2 D(x y x)=D(x y) x+x y D(x)+D(x) y x+x D(y x)
$$

for all $x, y \in R$, then $D$ is a derivation. Putting $x$ for $y$ in relations (1) and (2) leads to

$$
\begin{aligned}
D\left(x^{3}\right) & =D(x) x^{2}+x D(x) x+x^{2} D(x), \\
2 D\left(x^{3}\right) & =D\left(x^{2}\right) x+x^{2} D(x)+D(x) x^{2}+x D\left(x^{2}\right)
\end{aligned}
$$

for all $x \in R$. Recently, M. Fošner and the authors ([11]) proved the following result regarding the relation (4), which is related to the Vukman's conjecture mentioned above.

Theorem 2. Let $R$ be a 2-torsion free prime ring and let $D: R \rightarrow R$ be an additive mapping satisfying the relation

$$
2 D\left(x^{3}\right)=D\left(x^{2}\right) x+x^{2} D(x)+D(x) x^{2}+x D\left(x^{2}\right)
$$

for all $x \in R$. In this case $D$ is a derivation.

The relation (3) leads to the relation

$$
D\left(x^{n}\right)=\sum_{i=1}^{n} x^{i-1} D(x) x^{n-i},
$$

which was studied on prime rings by Beidar, M. Brešar, Chebotar and Martindale ([2]) (see also [15] for the result regarding the above relation on unital 
semiprime rings). It is our aim in this paper to obtain and study the relation, which generalizes the relation (4).

Putting $x, x^{2}, x^{3}, \ldots, x^{n-2}$ for $y$ in (2) leads to the following system of relations, respectively.

$$
\begin{aligned}
2 D\left(x^{3}\right) & =D\left(x^{2}\right) x+x^{2} D(x)+D(x) x^{2}+x D\left(x^{2}\right) \\
4 D\left(x^{4}\right) & =2 D\left(x^{3}\right) x+2 x^{3} D(x)+2 D(x) x^{3}+2 x D\left(x^{3}\right), \\
8 D\left(x^{5}\right) & =4 D\left(x^{4}\right) x+4 x^{4} D(x)+4 D(x) x^{4}+4 x D\left(x^{4}\right), \\
16 D\left(x^{6}\right)= & 8 D\left(x^{5}\right) x+8 x^{5} D(x)+8 D(x) x^{5}+8 x D\left(x^{5}\right), \\
& \vdots \\
2^{n-2} D\left(x^{n}\right)= & 2^{n-3} D\left(x^{n-1}\right) x+2^{n-3} x^{n-1} D(x)+2^{n-3} D(x) x^{n-1} \\
& +2^{n-3} x D\left(x^{n-1}\right) .
\end{aligned}
$$

Considering (5) in (6) leads to

$$
\begin{aligned}
4 D\left(x^{4}\right)= & 3 D(x) x^{3}+x D(x) x^{2}+x^{2} D(x) x+3 x^{3} D(x)+D\left(x^{2}\right) x^{2} \\
& +2 x D\left(x^{2}\right) x+x^{2} D\left(x^{2}\right) .
\end{aligned}
$$

Putting the above relation in the relation (7) we obtain

$$
\begin{aligned}
8 D\left(x^{5}\right)= & 7 D(x) x^{4}+4 x D(x) x^{3}+2 x^{2} D(x) x^{2}+4 x^{3} D(x) x+7 x^{4} D(x) \\
& +D\left(x^{2}\right) x^{3}+3 x D\left(x^{2}\right) x^{2}+3 x^{2} D\left(x^{2}\right) x+x^{3} D\left(x^{2}\right) .
\end{aligned}
$$

Considering the above relation in (8) we obtain

$$
\begin{aligned}
16 D\left(x^{6}\right)= & 15 D(x) x^{5}+11 x D(x) x^{4}+6 x^{2} D(x) x^{3}+6 x^{3} D(x) x^{2}+11 x^{4} D(x) x \\
& +15 x^{5} D(x)+D\left(x^{2}\right) x^{4}+4 x D\left(x^{2}\right) x^{3}+6 x^{2} D\left(x^{2}\right) x^{2}+4 x^{3} D\left(x^{2}\right) x \\
& +x^{4} D\left(x^{2}\right) .
\end{aligned}
$$

We see that in the above relations the coefficients of terms including $D\left(x^{2}\right)$ follow as $(1,1),(1,2,1),(1,3,3,1),(1,4,6,4,1)$ and therefore form a Pascal triangle. The coefficients of terms including $D(x)$ follow as $(1,0,1)$, $(3,1,1,3),(7,4,2,4,7),(15,11,6,6,11,15)$. Is there any specific algorithm that can foretell the coefficients of terms including $D(x)$ as we proceed with the above procedure? It turns out that the answer is positive. We will now present the results regarding the above speculations.

Pellicer and Alvo ([1]) delivered the following definition.

Definition 3. The modified Pascal triangle $P(m, n)$ generated by sequences $\left\{a_{m}\right\}$ and $\left\{b_{n}\right\}$ is determined by relations:

(i) $P(m, 0)=a_{m}$,

(ii) $P(0, n)=b_{n}$,

(iii) $P(m, n)=P(m, n-1)+P(m-1, n)$ 
for all $m, n \in \mathbb{N}$.

Below we can see the scheme of the modified Pascal triangle, which is generated by the sequences $\left\{a_{m}\right\}$ and $\left\{b_{n}\right\}$.

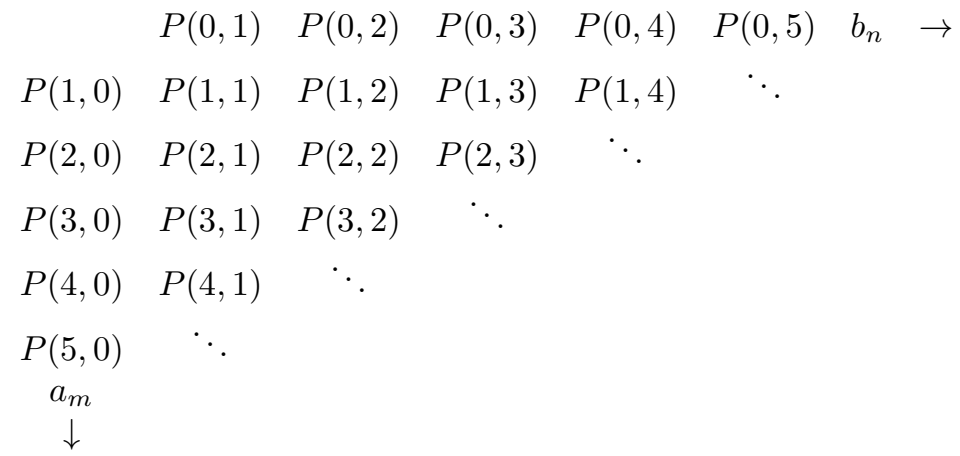

In the same paper Pellicer and Alvo proved the following theorem, which states that given the generating recursive additive pattern of a modified Pascal triangle and given the border sequences, the inside triangle is uniquely determined.

Theorem 4. Given a modified Pascal triangle $P$ it holds that

$$
P(m, n)=\sum_{k=1}^{m} a_{k}\left(\begin{array}{c}
m+n-k-1 \\
m-k
\end{array}\right)+\sum_{k=1}^{n} b_{k}\left(\begin{array}{c}
m+n-k-1 \\
n-k
\end{array}\right)
$$

for all $m, n \in \mathbb{N}$.

We can now proceed with the work regarding the system of relations (5), (6), (7), (8), (9). We have already mentioned that the coefficients for all terms including $D\left(x^{2}\right)$ form a Pascal triangle. According to the theory above, the coefficients for all terms including $D(x)$ form the following modified Pascal triangle, generated by the sequences

$$
\begin{aligned}
& \left\{a_{m}\right\}=\left\{b_{n}\right\}=\left(0,1,3,7,15, \ldots, 2^{i-1}-1, \ldots\right), i=1,2,3 \ldots \\
& \begin{array}{rrrrrrrr} 
& 0 & 1 & 3 & 7 & 15 & 31 & \cdots \\
0 & 0 & 1 & 4 & 11 & 26 & \ddots & \\
1 & 1 & 2 & 6 & 17 & \ddots & & \\
3 & 4 & 6 & 12 & \ddots & & & \\
7 & 11 & 17 & \ddots & & & & \\
15 & 26 & \ddots & & & & & \\
31 & \ddots & & & & & & \\
\vdots & & & & & & &
\end{array}
\end{aligned}
$$


According to Theorem 4, the induction and (9) lead to

$$
\begin{aligned}
2^{n-2} D\left(x^{n}\right) & \\
= & \sum_{i=0}^{n-2}\left(\begin{array}{c}
n-2 \\
i
\end{array}\right) x^{i} D\left(x^{2}\right) x^{n-2-i}+\sum_{i=0}^{n-1} P(i, n-1-i) x^{i} D(x) x^{n-1-i} \\
= & \sum_{i=0}^{n-2}\left(\begin{array}{c}
n-2 \\
i
\end{array}\right) x^{i} D\left(x^{2}\right) x^{n-2-i}+P(0, n-1) D(x) x^{n-1}+P(n-1,0) x^{n-1} D(x) \\
& +\sum_{i=1}^{n-2}\left(\sum_{k=1}^{i} a_{k}\left(\begin{array}{c}
i+n-1-i-k-1 \\
i-k
\end{array}\right)+\sum_{k=1}^{n-1-i} b_{k}\left(\begin{array}{c}
i+n-1-i-k-1 \\
n-1-i-k
\end{array}\right)\right) x^{i} D(x) x^{n-1-i} \\
= & \sum_{i=0}^{n-2}\left(\begin{array}{c}
n-2 \\
i
\end{array}\right) x^{i} D\left(x^{2}\right) x^{n-2-i}+\left(2^{n-2}-1\right)\left(D(x) x^{n-1}+x^{n-1} D(x)\right) \\
& +\sum_{i=1}^{n-2}\left(\sum_{k=1}^{i}\left(2^{k-1}-1\right)\left(\begin{array}{c}
n-k-2 \\
i-k
\end{array}\right)+\sum_{k=1}^{n-1-i}\left(2^{k-1}-1\right)\left(\begin{array}{c}
n-k-2 \\
n-i-k-1
\end{array}\right)\right) x^{i} D(x) x^{n-1-i} \\
= & \sum_{i=0}^{n-2}\left(\begin{array}{c}
n-2 \\
i
\end{array}\right) x^{i} D\left(x^{2}\right) x^{n-2-i}+\left(2^{n-2}-1\right)\left(D(x) x^{n-1}+x^{n-1} D(x)\right) \\
& +\sum_{i=1}^{n-2}\left(\sum_{k=2}^{i}\left(2^{k-1}-1\right)\left(\begin{array}{c}
n-k-2 \\
i-k
\end{array}\right)+\sum_{k=2}^{n-1-i}\left(2^{k-1}-1\right)\left(\begin{array}{c}
n-k-2 \\
n-i-k-1
\end{array}\right)\right) x^{i} D(x) x^{n-1-i} .
\end{aligned}
$$

Therefore

$$
\begin{aligned}
& 2^{n-2} D\left(x^{n}\right) \\
& =\sum_{i=0}^{n-2}\left(\begin{array}{c}
n-2 \\
i
\end{array}\right) x^{i} D\left(x^{2}\right) x^{n-2-i}+\left(2^{n-2}-1\right)\left(D(x) x^{n-1}+x^{n-1} D(x)\right) \\
& \quad+\sum_{i=1}^{n-2}\left(\sum_{k=2}^{i}\left(2^{k-1}-1\right)\left(\begin{array}{c}
n-k-2 \\
i-k
\end{array}\right)+\sum_{k=2}^{n-1-i}\left(2^{k-1}-1\right)\left(\begin{array}{c}
n-k-2 \\
n-i-k-1
\end{array}\right)\right) x^{i} D(x) x^{n-1-i} .
\end{aligned}
$$

Let us note that $\sum_{k=2}^{1} f(k)=0$, for any function $f$.

We have to remark that the additive solutions of $\sum_{i=0}^{n} c_{i} x^{i} D\left(x^{n-i}\right)=0$ for fixed constants $c_{i} \in R$, where $R$ is a commutative ring, was partially characterized by Ebanks ([7]) and Ebanks et al. ([8]). Later, the problem was fully solved by Gselmann et al. ([12]) and Ebanks ([9]) independently using different approach.

It is our aim in this paper to prove the following result.

Theorem 5. Let $n \geq 3$ be some fixed integer, let $R$ be $a(n+1) ! 2^{n-2}$ torsion free unital semiprime ring and let $D: R \rightarrow R$ be an additive mapping 
satisfying the relation

$$
\begin{aligned}
& 2^{n-2} D\left(x^{n}\right) \\
& =\sum_{i=0}^{n-2}\left(\begin{array}{c}
n-2 \\
i
\end{array}\right) x^{i} D\left(x^{2}\right) x^{n-2-i}+\left(2^{n-2}-1\right)\left(D(x) x^{n-1}+x^{n-1} D(x)\right) \\
& \quad+\sum_{i=1}^{n-2}\left(\sum_{k=2}^{i}\left(2^{k-1}-1\right)\left(\begin{array}{c}
n-k-2 \\
i-k
\end{array}\right)+\sum_{k=2}^{n-1-i}\left(2^{k-1}-1\right)\left(\begin{array}{c}
n-k-2 \\
n-i-k-1
\end{array}\right)\right) x^{i} D(x) x^{n-1-i}
\end{aligned}
$$

for all $x \in R$. In this case $D$ is a derivation.

For the seek of completeness, we may mention that for commutative, $(n+1) ! 2^{n-2}$-torsion free rings the above equation can be reduced to $(n-$ 2) $x^{n-1} D(x)+x^{n-2} D\left(x^{2}\right)-D\left(x^{n}\right)=0$. By [8], $D$ has to be a derivation of order at most 2. Since every derivation (of order 1 ) is clearly a solution of this equation it is enough to verify that the equation for derivations of order 2 does not hold which can be checked by direct calculation.

For the proof of the above theorem, we will need the following lemma.

Lemma 6. The relation

$$
\sum_{i=2}^{n-2} \sum_{k=2}^{i}\left(2^{k-1}-1\right)\left(\begin{array}{c}
n-k-2 \\
i-k
\end{array}\right)=(n-4) 2^{n-3}+1
$$

holds for all integers $n \geq 3$.

Proof. Considering $\sum_{k=0}^{n}\left(\begin{array}{l}n \\ k\end{array}\right)=2^{n}$ in the next calculation proves the lemma.

$$
\begin{aligned}
\sum_{i=2}^{n-2} & \sum_{k=2}^{i}\left(2^{k-1}-1\right)\left(\begin{array}{c}
n-k-2 \\
i-k
\end{array}\right) \\
= & \sum_{k=2}^{2}\left(2^{k-1}-1\right)\left(\begin{array}{c}
n-k-2 \\
2-k
\end{array}\right)+\sum_{k=2}^{3}\left(2^{k-1}-1\right)\left(\begin{array}{c}
n-k-2 \\
3-k
\end{array}\right)+\cdots \\
& +\sum_{k=2}^{n-3}\left(2^{k-1}-1\right)\left(\begin{array}{c}
n-k-2 \\
n-k-3
\end{array}\right)+\sum_{k=2}^{n-2}\left(2^{k-1}-1\right)\left(\begin{array}{c}
n-k-2 \\
n-k-2
\end{array}\right) \\
= & \left(2^{1}-1\right)\left(\left(\begin{array}{c}
n-4 \\
0
\end{array}\right)+\left(\begin{array}{c}
n-4 \\
1
\end{array}\right)+\cdots+\left(\begin{array}{c}
n-4 \\
n-4
\end{array}\right)\right) \\
& +\left(2^{2}-1\right)\left(\left(\begin{array}{c}
n-5 \\
0
\end{array}\right)+\left(\begin{array}{c}
n-5 \\
1
\end{array}\right)+\cdots+\left(\begin{array}{c}
n-5 \\
n-5
\end{array}\right)\right)+\cdots \\
& +\left(2^{n-4}-1\right)\left(\left(\begin{array}{c}
1 \\
0
\end{array}\right)+\left(\begin{array}{c}
1 \\
1
\end{array}\right)\right)+\left(2^{n-3}-1\right)\left(\begin{array}{l}
0 \\
0
\end{array}\right) \\
= & (2-1) 2^{n-4}+\left(2^{2}-1\right) 2^{n-5}+\cdots+\left(2^{n-4}-1\right) 2^{1}+\left(2^{n-3}-1\right) 2^{0} \\
= & (n-3) 2^{n-3}-\left(1+2+2^{2}+2^{3}+\cdots+2^{n-5}+2^{n-4}\right) \\
= & (n-3) 2^{n-3}-\left(2^{n-3}-1\right)
\end{aligned}
$$


$=(n-4) 2^{n-3}+1$.

We are now in the position to prove Theorem 5 .

Proof of TheOrem 5. We have the relation

(10)

$$
\begin{aligned}
& 2^{n-2} D\left(x^{n}\right) \\
& =\sum_{i=0}^{n-2}\left(\begin{array}{c}
n-2 \\
i
\end{array}\right) x^{i} D\left(x^{2}\right) x^{n-2-i}+\left(2^{n-2}-1\right)\left(D(x) x^{n-1}+x^{n-1} D(x)\right) \\
& \quad+\sum_{i=1}^{n-2}\left(\sum_{k=2}^{i}\left(2^{k-1}-1\right)\left(\begin{array}{c}
n-k-2 \\
i-k
\end{array}\right)+\sum_{k=2}^{n-1-i}\left(2^{k-1}-1\right)\left(\begin{array}{c}
n-k-2 \\
n-i-k-1
\end{array}\right)\right) x^{i} D(x) x^{n-1-i} .
\end{aligned}
$$

We will denote the identity element of the ring $R$ by $e$. Putting $e$ for $x$ in the above relation we obtain

$$
\begin{aligned}
2^{n-2} D(e)= & \sum_{i=0}^{n-2}\left(\begin{array}{c}
n-2 \\
i
\end{array}\right) D(e)+2\left(2^{n-2}-1\right) D(e) \\
& +2\left(\sum_{k=2}^{2}\left(2^{k-1}-1\right)\left(\begin{array}{c}
n-k-2 \\
2-k
\end{array}\right)+\sum_{k=2}^{3}\left(2^{k-1}-1\right)\left(\begin{array}{c}
n-k-2 \\
3-k
\end{array}\right)+\cdots\right. \\
& \left.+\sum_{k=2}^{n-3}\left(2^{k-1}-1\right)\left(\begin{array}{c}
n-k-2 \\
n-k-3
\end{array}\right)+\sum_{k=2}^{n-2}\left(2^{k-1}-1\right)\left(\begin{array}{c}
n-k-2 \\
n-k-2
\end{array}\right)\right) D(e) .
\end{aligned}
$$

Lemma 6 reduces the above relation to

$$
2^{n-2} D(e)=2^{n-2} D(e)+2\left(2^{n-2}-1\right) D(e)+2\left((n-4) 2^{n-3}+1\right) D(e) .
$$

Further calculation leads to

$$
(n-2) 2^{n-2} D(e)=0
$$

and considering the torsion freeness, we obtain

$$
D(e)=0 .
$$

Let $y$ be an arbitrary element from $Z(R)$. Putting $x+y$ for $x$ in the relation (10) we obtain

$$
\begin{aligned}
& 2^{n-2} \sum_{i=0}^{n}\left(\begin{array}{c}
n \\
i
\end{array}\right) D\left(x^{n-i} y^{i}\right) \\
& =\left(\begin{array}{c}
n-2 \\
0
\end{array}\right) D\left(x^{2}+2 x y+y^{2}\right)\left(\sum_{i=0}^{n-2}\left(\begin{array}{c}
n-2 \\
i
\end{array}\right) x^{n-2-i} y^{i}\right) \\
& \quad+\left(\begin{array}{c}
n-2 \\
1
\end{array}\right)(x+y) D\left(x^{2}+2 x y+y^{2}\right)\left(\sum_{i=0}^{n-3}\left(\begin{array}{c}
n-3 \\
i
\end{array}\right) x^{n-3-i} y^{i}\right)
\end{aligned}
$$




$$
\begin{aligned}
& +\cdots \\
& +\left(\begin{array}{c}
n-2 \\
n-3
\end{array}\right)\left(\sum_{i=0}^{n-3}\left(\begin{array}{c}
n-3 \\
i
\end{array}\right) x^{n-3-i} y^{i}\right) D\left(x^{2}+2 x y+y^{2}\right)(x+y) \\
& +\left(\begin{array}{c}
n-2 \\
n-2
\end{array}\right)\left(\sum_{i=0}^{n-2}\left(\begin{array}{c}
n-2 \\
i
\end{array}\right) x^{n-2-i} y^{i}\right) D\left(x^{2}+2 x y+y^{2}\right) \\
& +\left(2^{n-2}-1\right) \sum_{i=0}^{n-1}\left(\begin{array}{c}
n-1 \\
i
\end{array}\right)\left(D(x+y) x^{n-1-i} y^{i}+x^{n-1-i} y^{i} D(x+y)\right) \\
& +\left(\sum_{k=2}^{1}\left(2^{k-1}-1\right)\left(\begin{array}{c}
n-k-2 \\
1-k
\end{array}\right)+\sum_{k=2}^{n-2}\left(2^{k-1}-1\right)\left(\begin{array}{l}
n-k-2 \\
n-k-2
\end{array}\right)\right) \\
& \cdot(x+y) D(x+y)\left(\sum_{j=0}^{n-2}\left(\begin{array}{c}
n-2 \\
j
\end{array}\right) x^{n-2-j} y^{j}\right) \\
& \left(\sum_{k=2}^{2}\left(2^{k-1}-1\right)\left(\begin{array}{c}
n-k-2 \\
2-k
\end{array}\right)+\sum_{k=2}^{n-3}\left(2^{k-1}-1\right)\left(\begin{array}{c}
n-k-2 \\
n-k-3
\end{array}\right)\right) \\
& \cdot\left(x^{2}+2 x y+y^{2}\right) D(x+y)\left(\sum_{j=0}^{n-3}\left(\begin{array}{c}
n-3 \\
j
\end{array}\right) x^{n-3-j} y^{j}\right) \\
& +\cdots \\
& +\left(\sum_{k=2}^{n-3}\left(2^{k-1}-1\right)\left(\begin{array}{c}
n-k-2 \\
n-k-3
\end{array}\right)+\sum_{k=2}^{2}\left(2^{k-1}-1\right)\left(\begin{array}{c}
n-k-2 \\
2-k
\end{array}\right)\right) \\
& \cdot\left(\sum_{j=0}^{n-3}\left(\begin{array}{c}
n-3 \\
j
\end{array}\right) x^{n-3-j} y^{j}\right) D(x+y)\left(x^{2}+2 x y+y^{2}\right) \\
& +\left(\sum_{k=2}^{n-2}\left(2^{k-1}-1\right)\left(\begin{array}{c}
n-k-2 \\
n-k-2
\end{array}\right)+\sum_{k=2}^{1}\left(2^{k-1}-1\right)\left(\begin{array}{c}
n-k-2 \\
1-k
\end{array}\right)\right) \\
& \cdot\left(\sum_{j=0}^{n-2}\left(\begin{array}{c}
n-2 \\
j
\end{array}\right) x^{n-2-j} y^{j}\right) D(x+y)(x+y) .
\end{aligned}
$$

Using (10) and rearranging the above relation in sense of collecting together terms involving equal number of factors of $y$, we obtain

$$
\sum_{i=1}^{n-1} f_{i}(x, y)=0
$$


where $f_{i}(x, y)$ stands for the expression of terms involving $i$ factors of $y$. Replacing $x$ by $x+2 y, x+3 y, \ldots, x+(n-1) y$ in turn in the relation (10) and expressing the resulting system of $n-1$ homogeneous equations of variables $f_{i}(x, y), i=1,2, \ldots, n-1$, we see that the coefficient matrix of the system is a Vandermonde matrix

$$
\left[\begin{array}{cccc}
1 & 1 & \cdots & 1 \\
2 & 2^{2} & \cdots & 2^{n-1} \\
\vdots & \vdots & \ddots & \vdots \\
n-1 & (n-1)^{2} & \cdots & (n-1)^{n-1}
\end{array}\right]
$$

Since the determinant of the matrix is different from zero, it follows that the system has only a trivial solution. In particular, if $y$ is replaced with the identity element $e$ and considering (11), we obtain

$$
\begin{aligned}
& f_{n-2}(x, e) \\
& =2^{n-2}\left(\begin{array}{c}
n \\
n-2
\end{array}\right) D\left(x^{2}\right)-\left(\begin{array}{c}
n-2 \\
0
\end{array}\right)\left(\left(\begin{array}{c}
n-2 \\
n-2
\end{array}\right) D\left(x^{2}\right)+2\left(\begin{array}{c}
n-2 \\
n-3
\end{array}\right) D(x) x\right) \\
& \text { - }\left(\begin{array}{c}
n-2 \\
1
\end{array}\right)\left(\left(\begin{array}{c}
n-3 \\
n-3
\end{array}\right) D\left(x^{2}\right)+2\left(\begin{array}{c}
n-3 \\
n-4
\end{array}\right) D(x) x+2\left(\begin{array}{c}
1 \\
0
\end{array}\right) x D(x)\right) \\
& -\left(\begin{array}{c}
n-2 \\
2
\end{array}\right)\left(\left(\begin{array}{l}
n-4 \\
n-4
\end{array}\right) D\left(x^{2}\right)+2\left(\begin{array}{l}
n-4 \\
n-5
\end{array}\right) D(x) x+2\left(\begin{array}{l}
2 \\
1
\end{array}\right) x D(x)\right) \\
& -\cdots \text {. } \\
& -\left(\begin{array}{c}
n-2 \\
n-4
\end{array}\right)\left(\left(\begin{array}{l}
2 \\
2
\end{array}\right) D\left(x^{2}\right)+2\left(\begin{array}{l}
2 \\
1
\end{array}\right) D(x) x+2\left(\begin{array}{c}
n-4 \\
n-5
\end{array}\right) x D(x)\right) \\
& -\left(\begin{array}{l}
n-2 \\
n-3
\end{array}\right)\left(\left(\begin{array}{l}
1 \\
1
\end{array}\right) D\left(x^{2}\right)+2\left(\begin{array}{l}
1 \\
0
\end{array}\right) D(x) x+2\left(\begin{array}{l}
n-3 \\
n-4
\end{array}\right) x D(x)\right) \\
& -\left(\begin{array}{l}
n-2 \\
n-2
\end{array}\right)\left(\left(\begin{array}{l}
2 \\
2
\end{array}\right) D\left(x^{2}\right)+2\left(\begin{array}{l}
n-2 \\
n-3
\end{array}\right) x D(x)\right) \\
& -\left(2^{n-2}-1\right)\left(\begin{array}{c}
n-1 \\
n-2
\end{array}\right)(D(x) x+x D(x)) \\
& -\left(\sum_{k=2}^{1}\left(2^{k-1}-1\right)\left(\begin{array}{c}
n-k-2 \\
1-k
\end{array}\right)+\sum_{k=2}^{n-2}\left(2^{k-1}-1\right)\left(\begin{array}{c}
n-k-2 \\
n-k-2
\end{array}\right)\right) \\
& \cdot\left(\left(\begin{array}{l}
n-2 \\
n-3
\end{array}\right) D(x) x+\left(\begin{array}{l}
1 \\
0
\end{array}\right) x D(x)\right) \\
& -\left(\sum_{k=2}^{2}\left(2^{k-1}-1\right)\left(\begin{array}{c}
n-k-2 \\
2-k
\end{array}\right)+\sum_{k=2}^{n-3}\left(2^{k-1}-1\right)\left(\begin{array}{c}
n-k-2 \\
n-k-3
\end{array}\right)\right) \\
& \cdot\left(\left(\begin{array}{l}
n-3 \\
n-4
\end{array}\right) D(x) x+\left(\begin{array}{l}
2 \\
1
\end{array}\right) x D(x)\right) \\
& -\left(\sum_{k=2}^{3}\left(2^{k-1}-1\right)\left(\begin{array}{c}
n-k-2 \\
3-k
\end{array}\right)+\sum_{k=2}^{n-4}\left(2^{k-1}-1\right)\left(\begin{array}{c}
n-k-2 \\
n-k-4
\end{array}\right)\right) \\
& \cdot\left(\left(\begin{array}{l}
n-4 \\
n-5
\end{array}\right) D(x) x+\left(\begin{array}{l}
3 \\
2
\end{array}\right) x D(x)\right) \\
& -\cdots
\end{aligned}
$$




$$
\begin{aligned}
- & \left(\sum_{k=2}^{n-4}\left(2^{k-1}-1\right)\left(\begin{array}{l}
n-k-2 \\
n-k-4
\end{array}\right)+\sum_{k=2}^{3}\left(2^{k-1}-1\right)\left(\begin{array}{c}
n-k-2 \\
3-k
\end{array}\right)\right) \\
& \cdot\left(\left(\begin{array}{l}
3 \\
2
\end{array}\right) D(x) x+\left(\begin{array}{c}
n-4 \\
n-5
\end{array}\right) x D(x)\right) \\
- & \left(\sum_{k=2}^{n-3}\left(2^{k-1}-1\right)\left(\begin{array}{c}
n-k-2 \\
n-k-3
\end{array}\right)+\sum_{k=2}^{2}\left(2^{k-1}-1\right)\left(\begin{array}{c}
n-k-2 \\
2-k
\end{array}\right)\right) \\
& \left.\cdot\left(\begin{array}{l}
2 \\
1
\end{array}\right) D(x) x+\left(\begin{array}{c}
n-3 \\
n-4
\end{array}\right) x D(x)\right) \\
- & \left(\sum_{k=2}^{n-2}\left(2^{k-1}-1\right)\left(\begin{array}{l}
n-k-2 \\
n-k-2
\end{array}\right)+\sum_{k=2}^{1}\left(2^{k-1}-1\right)\left(\begin{array}{c}
n-k-2 \\
1-k
\end{array}\right)\right) \\
& \cdot\left(\left(\begin{array}{l}
1 \\
0
\end{array}\right) D(x) x+\left(\begin{array}{c}
n-2 \\
n-3
\end{array}\right) x D(x)\right) .
\end{aligned}
$$

As the sums in the above relation are pairwise equal, the above relation reduces to

$$
\begin{aligned}
& 2^{n-2}\left(\begin{array}{c}
n \\
n-2
\end{array}\right) D\left(x^{2}\right) \\
&= 2^{n-2} D\left(x^{2}\right) \\
&+2\left(\left(\begin{array}{c}
n-2 \\
0
\end{array}\right)(n-2)+\left(\begin{array}{c}
n-2 \\
1
\end{array}\right)(n-3)+\cdots+\left(\begin{array}{c}
n-2 \\
n-4
\end{array}\right) 2+\left(\begin{array}{c}
n-2 \\
n-3
\end{array}\right)\right) \\
& \cdot(D(x) x+x D(x)) \\
&+\left(2^{n-2}-1\right)(n-1)(D(x) x+x D(x)) \\
&+(n-1)\left(\sum_{k=2}^{1}\left(2^{k-1}-1\right)\left(\begin{array}{c}
n-k-2 \\
1-k
\end{array}\right)+\sum_{k=2}^{2}\left(2^{k-1}-1\right)\left(\begin{array}{c}
n-k-2 \\
2-k
\end{array}\right)\right. \\
&+\cdots \\
&\left.+\sum_{k=2}^{n-3}\left(2^{k-1}-1\right)\left(\begin{array}{c}
n-k-2 \\
n-k-3
\end{array}\right)+\sum_{k=2}^{n-2}\left(2^{k-1}-1\right)\left(\begin{array}{c}
n-k-2 \\
n-k-2
\end{array}\right)\right)(D(x) x+x D(x)) .
\end{aligned}
$$

Using Lemma 6 in the above relation and some calculation leads to

$$
\begin{aligned}
& 2^{n-2}\left(\left(\begin{array}{c}
n \\
n-2
\end{array}\right)-1\right) D\left(x^{2}\right) \\
& =2 \sum_{k=0}^{n-3}\left(\begin{array}{c}
n-2 \\
k
\end{array}\right)(n-2-k)(D(x) x+x D(x)) \\
& \quad+(n-1)\left(2^{n-2}-1\right)(D(x) x+x D(x)) \\
& \quad+(n-1)\left((n-4) 2^{n-3}+1\right)(D(x) x+x D(x)) .
\end{aligned}
$$


Since

$$
\begin{aligned}
\sum_{k=0}^{n-3}\left(\begin{array}{c}
n-2 \\
k
\end{array}\right)(n-2-k) & =\sum_{k=0}^{n-2}\left(\begin{array}{c}
n-2 \\
k
\end{array}\right)(n-2-k) \\
& =\sum_{k=0}^{n-2}\left(\begin{array}{c}
n-2 \\
k
\end{array}\right)(n-2)-\sum_{k=0}^{n-2}\left(\begin{array}{c}
n-2 \\
k
\end{array}\right) k \\
& =(n-2) 2^{n-2}-(n-2) 2^{n-3}=(n-2) 2^{n-3},
\end{aligned}
$$

the relation (12) can be rewritten as

$$
\begin{aligned}
& 2^{n-3}(n+1)(n-2) D\left(x^{2}\right) \\
& =\left((n-2) 2^{n-2}+(n-1)\left(2^{n-2}+(n-4) 2^{n-3}\right)\right)(D(x) x+x D(x)) \\
& =\left((n-2) 2^{n-2}+(n-1)(n-2) 2^{n-3}\right)(D(x) x+x D(x)) \\
& =2^{n-3}(n+1)(n-2)(D(x) x+x D(x)) .
\end{aligned}
$$

We therefore have

$$
2^{n-3}(n+1)(n-2) D\left(x^{2}\right)=2^{n-3}(n+1)(n-2)(D(x) x+x D(x)) .
$$

Since $R$ is $(n+1) ! 2^{n-2}$-torsion free, the above relation reduces to

$$
D\left(x^{2}\right)=D(x) x+x D(x)
$$

for all $x \in R$. In other words, $D$ is a Jordan derivation on $R$. According to Cusack's generalization of Herstein theorem, one can conclude that $D$ is a derivation, which completes the proof of the theorem.

\section{REFERENCES}

[1] D. Alvo and R. Pellicer, Modified Pascal triangle and Pascal surfaces, http://www. academia.edu/956605.

[2] K. I. Beidar, M. Brešar, M. A. Chebotar and W. S. Martindale 3rd, On Herstein's Lie map conjectures II, J. Algebra 238 (2001), 239-264.

[3] M. Brešar and J. Vukman, Jordan derivations on prime rings, Bull. Austral. Math. Soc. 37 (1988), 321-322.

[4] M. Brešar, Jordan derivations on semiprime rings, Proc. Amer. Math. Soc. 104 (1988), 1003-1006.

[5] M. Brešar, Jordan mappings of semiprime rings, J. Algebra 127 (1989), 218-228.

[6] J. Cusack, Jordan derivations on rings, Proc. Amer. Math. Soc. 53 (1975), 321-324.

[7] B. Ebanks, Characterizing ring derivations of all orders via functional equations: results and open problems, Aequationes Math. 89 (2015), 685-718.

[8] B. Ebanks, T. Riedel and P. K. Sahoo, On the order of a derivation, Aequationes Math. 90 (2016), 335-340.

[9] B. Ebanks, Functional equations characterizing derivations: a synthesis, Results Math. 73 (2018), No. 120, 13 pp.

[10] D. Eremita, I. Kosi-Ulbl and J. Vukman, On certain equations in rings, Bull. Austral. Math. Soc. 71 (2005), 53-60.

[11] M. Fošner, N. Širovnik and J.Vukman, A result related to Herstein theorem, Bull. Malays. Math. Sci. Soc. 39 (2016), 885-899. 
[12] E. Gselmann, G. Kiss and Cs. Vincze, On functional equations characterizing derivations: methods and examples, Results Math. 73 (2018), No. 74, 27 pp.

[13] I. N. Herstein, Jordan derivations of prime rings, Proc. Amer. Math. Soc. 8 (1957), $1104-1120$.

[14] I. Kosi-Ulbl and J. Vukman, An equation related to centralizers in semiprime rings, Glas. Mat. Ser. III 38(58) (2003), 253-261.

[15] I. Kosi-Ulbl and J. Vukman, A note on derivations in semiprime rings, Int. J. Math. Math. Sci. 20 (2005), 3347-3350.

[16] C.-K. Liu and W.-K. Shiue, Generalized Jordan triple $(\theta, \phi)$-derivations on semiprime rings, Taiwanese J. Math. 11 (2007), 1397-1406.

[17] J. Vukman, Centralizers on semiprime rings, Comment. Math. Univ. Carolin. 42 (2001), 237-245.

[18] J. Vukman, Some remarks on derivations in semiprime rings and standard operator algebras, Glas. Mat. Ser. III 46(66) (2011), 43-48.

I. Kosi-Ulbl

Faculty of Mechanical Engineering

University of Maribor

Smetanova 17, 2000 Maribor

Slovenia

E-mail: irena.kosi@um.si

N. Širovnik

WinSystems, Global Gaming Solutions Partner

E-mail: nejc.sirovnik@gmail.com

J. Vukman

Faculty of Natural Sciences and Mathematics

Department of Mathematics and Computer Science

University of Maribor

Koroška 160, 2000 Maribor

Slovenia

E-mail: joso.vukman@guest.um.si

Received: 20.5.2020. 